\title{
QUARENTA E OITO ANOS DE MELHORAMENTO DA VIDEIRA EM SÃO PAULO, BRASIL ${ }^{1}$
}

\author{
C.P. FERRI'; C.V. POMMER ${ }^{3}$ \\ ${ }^{2}$ Engenheira Agrônoma com Bolsa de Aperfeiçoamento da FAPESP \\ ${ }^{3}$ Instituto Agronômico de Campinas, C.P. 28, CEP: 13001-970-Campinas,SP
}

\begin{abstract}
RESUMO: A cultura de uvas de mesa em São Paulo vem aumentando de importância, o que é flagrante pelos incrementos na área plantada verificados nos ultimos anos. A demanda por tecnologia tem acompanhado esse crescimento, especialmente no que se refere a novas variedades que atendam tanto ao mercado externo, quanto ao interno, com uvas de alta qualidade. No programa de melhoramento para uvas de mesa no Instituto Agronômico, iniciado em 1943, praticamente nenhum estudo foi efetuado sobre os progenitores utilizados nos cruzamentos. Neste trabalho foram avaliados centenas de cruzamentos e as variedades envolvidas, bem como suas progênies e seu interrelacionamento genealogico. De acordo com os dados analisados e os parâmetros utilizados, foram considerados preferenciais num programa de cruzamento para uvas de mesa as seguintes variedades: 'Italia', 'Patricia', 'Soraya', 'Cardinal','Roberta', 'Maria Rosa', 'Carolina', 'Moscatel de Hamburgo', 'Ligia', 'Angelina', 'Marilia', 'Alphonse Lavallée', 'Traviú', 'Ezequiel', 'Grão Mogol', 'Yole', 'Geni', 'A Dona', 'Aurora', 'Piratininga', 'Maria', 'Iracema'. Descritores: videira, melhoramento da videira, progenitores, seleção
\end{abstract}

\section{FORTY EIGTH YEARS OF GRAPE BREEDING IN SÃO PAULO, BRAZIL}

\begin{abstract}
The importance of table grape cultivation in São Paulo State, Brazil, is increasing in the last years, which is demonstrated by its acreage increase. Demands for technology improvements have followed, specially in respect to the development of new cultivars that can produce high quality grapes for exportation or internal markets purposes. Since the beginning of the grape breeding program at the Instituto Agronomico de Campinas (IAC) in 1943, no study was carried out on the parents used in crossings. In this study, hundreds of crossings, parents, as well as resulting progenies and their genealogical (pedigree) relationship were evaluated. According to the analysed data and parameters used we may consider as preferential the following cultivars: 'Italia', 'Patricia', 'Soraya', 'Cardinal','Roberta', 'Maria Rosa', 'Carolina', 'Moscatel de Hamburgo', 'Ligia', 'Angelina', 'Marlia', 'Alphonse Lavallé', 'Traviú', 'Ezequiel', 'Grāo Mogol', 'Yole', 'Geni', 'A Dona', 'Aurora', 'Piratininga', 'Maria', 'Iracema'. Key Words: grapes, grape breeding, parents, selection
\end{abstract}

\section{INTRODUÇÃO}

A viticultura no Brasil ocupa pouco mais de 60.000 hectares concentrados nos estados do Rio Grande do Sul $(40.000 \mathrm{ha})$, São Paulo (10.000 ha), Santa Catarina (4.700 ha), Paraná (2.700 ha), Pernambuco (1.300 ha), Bahia (800 ha) e Minas Gerais ( 800 ha).

A área com videira em São Paulo manteve-se estável ao redor de 8.000 hectares até 1988 mas nos últimos anos cresceu sensivelmente elevando-se para cerca de 10.000 hectares.

A relevância da viticultura em São Paulo exigiu o desenvolvimento de amplo programa de melhoramento genético no Instituto Agronômico de Campinas, iniciado em 1943 e ativo até hoje (SANTOS NETO, 1955 e 1969; POMMER, 1993). No princípio, o programa visava a obtenção de variedades de uvas de vinho, uvas de mesa e portaenxerto (SANTOS NETO, 1971), mas com o decréscimo na importância da indústria vinícola no Estado, a busca por melhores variedades de uva de mesa passou a predominar.

Um programa de melhoramento genético que tenha por objetivo a obtenção de tais variedades depende, em alto grau, de bons progenitores cujas qualidades devem ser conhecidas para destacá-los entre centenas de variedades existentes.

1 Trabalho realizado com auxílio da FAPESP

Sci. agric., Piracicaba, 52(1):107-122, jan./abr. 1995 
No Instituto Agronômico de Campinas mais de 2.000 cruzamentos foram feitos em quase $\mathbf{5 0}$ anos de trabalho, utilizando mais de 800 progenitores.

$O$ objetivo do presente trabalho é o de estudar o emprego desses progenitores no IAC e seu interrelacionamento nas progênies obtidas, para conhecimento de seu potencial e de sua efetiva contribuição no melhoramento da videira (TABELA 1).

\section{MATERIAL E MÉTODOS}

As variedades e as espécies, a maioria resultante de introduções de outras regiōes e/ou países, destacavam-se por alguma qualidade e eram utilizadas conforme sua finalidade (mesa, vinho ou porta-enxertos). No caso específico de uvas para vinho, as primeiras introduções foram feitas por Dafert (DAFERT \& LEHMANN, 1895), constituídas por viníferas e híbridos obtidos na França. As espécies americanas, por seu lado, foram introduzidas a partir de recomendações de Olmo (SOUSA, 1969).

$\mathrm{Na}$ medida em que foram obtidos os primeiros resultados, com o surgimento de bons híbridos estes passaram a ser utilizados nos cruzamentos conforme apresentassem características de destaque. Com o passar dos anos, variedades surgidas no mundo todo puderam ser introduzidas no programa, especialmente nos últimos 10 anos.

A metodologia utilizada para os principais tópicos estudados foi a seguinte:

a) Frequência de utilização de variedades e/ou espécies.

Fez-se a catalogação dos dados no computador via software DBase III Plus, que permitiu no final a recuperação e a consolidação das informações. Dependendo dos dados, foram feitos agrupamentos por fases de programa (mesa, vinho, porta-enxerto). Gráficos auxiliaram na análise e manuseio dos dados.

b) Genealogia das variedades lançadas.

Ao longo dos 48 anos, mais de 40 variedades foram lançadas ou, pelo menos, receberam a denominação de variedade. Com a elaboração de fichários apropriados, foi recuperada a informação sobre a genealogia completa dessas variedades, com o auxílio de publicações básicas (HEDRICK, 1908; SOUSA, 1959; RIBAS, 1973; CAMARGO \& DIAS, 1986a e b). c) Estimativa de coeficientes de endogamia.

A adequada tabulação dos dados permitiu o cálculo de estimativas de coeficientes de endogamia, pelo menos entre as principais variedades utilizadas no programa e as variedades lançadas. O agrupamento por espécie permitiu o cálculo da proporção teórica de transmissão de fatores genéticos (POMMER \& BASTOS, 1984) para conhecimento da real contribuição das espécies de Vitis envolvidas em programas tão extensos.

d) Avaliação das principais progênies e do índice de sobrevivência.

Com os dados básicos anotados sobre cada cruzamento, obtiveram-se informações que permitiram a identificação de algumas progênies, entre as tantas obtidas, que se destacaram em alguma característica. Confrontaram-se essas informações nos diferentes anos e nos diferentes cruzamentos para a detecção de material genético (variedades, clones e espécies) com maior capacidade de combinação.

Da mesma forma, para certa parte dos cruzamentos há informação sobre o número de sementes obtidas, o número de sementes germinadas, o número de plantas transplantadas no campo e o número de plantas definitivamente estabelecidas (desenvolvidas). Avaliaram-se estes dados para chegar aos "índices de sobrevivência".

e) Seleção de progenitores.

A análise conjunta de todos os estudos descritos em a, b, c, d levou ao objetivo central que a pesquisa permitiu a seleção de progenitores.

\section{RESULTADOS E DISCUSSÃO}

Os resultados ao longo dos 48 anos foram divididos didaticamente em períodos de 8 anos para melhor destacá-los:

período 1: 1943 a 1950 - 318 cruzamentos período 2: 1951 a 1958 - 518 cruzamentos periodo 3: 1959 a 1966 - 478 cruzamentos período 4: 1967 a 1974 - 519 cruzamentos período 5: 1975 a 1982 - 316 cruzamentos período 6: 1983 a 1990 - 269 cruzamentos

Percebe-se que o período de maior atividade, em número de cruzamentos, foi de 1951 a 1974, quando foram efetuados 1.515 dos 2.418 cruzamentos. Apenas 52 autofecundações foram feitas nos 48 anos estudados. 
TABELA 1 - Variedades de videiras utilizadaos no programa de melhoramento do IAC, de 1943 a 1990 , com seus respectivos números de identificação $(\mathrm{N})$.

\begin{tabular}{|c|c|c|c|c|c|}
\hline $\mathbf{N}$ & VARIEDADE & $\mathrm{N}$ & VARIEDADE & $\mathrm{N}$ & VARIEDADE \\
\hline 1 & 59-D10 & 50 & BONARDA & 99 & GOLDEN NOIR \\
\hline 2 & 62-D10 & 51 & BR 420-A & 100 & GOLDEN QUEEN \\
\hline 3 & 414 & 52 & BRANCA DA CALÁBRIA & 101 & GREC ROUGE \\
\hline 4 & 432-D10 & 53 & BURGUNDER KASTENHOLTZ & 102 & GROIA \\
\hline 5 & 449-D8 & 54 & C.17-115 & 103 & GROS COLMAN \\
\hline 6 & 493-D10 & 55 & C.21-205 & 104 & HARTFORD \\
\hline 7 & 577 & 56 & C. $24-39$ & 105 & HERBEMONT \\
\hline 8 & 4308-5 & 57 & C.33-199 & 106 & HIGHLAND \\
\hline 9 & $4313-12$ & 58 & C.39-65 & 107 & HUBARD \\
\hline 10 & $4416-2$ & 59 & C. $49-190$ & 108 & IAC $1 / 78$ \\
\hline 11 & $4421-1$ & 60 & C. $54-123$ & & até \\
\hline 12 & $4421-4$ & 61 & C. $55-132$ & 734 & IAC $1899-7$ \\
\hline 13 & $4421-10$ & 62 & C. $55-152$ & 735 & ISABELLA \\
\hline 14 & $4423-2$ & 63 & C. $68-154$ & 736 & TTALIA \\
\hline 15 & $4432-6$ & 64 & C. $68-174$ & 737 & IVES SEEDLING \\
\hline 16 & $4436-5$ & 65 & C.81-109 & 738 & JD 874 \\
\hline 17 & $5012-34$ & 66 & CABERNET SAUVIGNON & 739 & JD 930 \\
\hline 18 & $46160-2$ & 67 & CARDINAL & 740 & JEAN MATHIAS \\
\hline 19 & $46164-20$ & 68 & CARIGNANE & 741 & JUMBO \\
\hline 20 & $46181-2$ & 69 & CARMAN & 742 & KYOHO \\
\hline 21 & A.8-158 & 70 & CARMENERE & 743 & LIBERTY \\
\hline 22 & A.28-23 & 71 & CATAWBA ROSA & 744 & LINDLEY \\
\hline 23 & A.41-30 & 72 & CHAOUCH & 745 & LÜBECK \\
\hline 24 & A. $41-130$ & 73 & CHASSELAS DORÉ & 746 & MADRESFIELD COURT \\
\hline 25 & A. $59-72$ & 74 & CHENIN & 747 & MALASIA DE FURMANN \\
\hline 26 & A.81-79 & 75 & CHENIN BLANC & 748 & MALÈGUE \\
\hline 27 & A.92-197 & 76 & CHILE NORMANHA MOSCATO & 749 & MALÈGUE 1.64718 \\
\hline 28 & A.97-27 & 77 & CONCORD & 750 & MARAVILHA DE MALAGA \\
\hline 29 & A.97-68 & 78 & COUDERC & 751 & MERLOT \\
\hline 30 & ALIGOTÉ & 79 & COUDERC 16 & 752 & MEXICANA \\
\hline 31 & ALPHONSELAVALÉE & 80 & COUDERC 17 & 753 & MEXICANA-3 \\
\hline 32 & ALVARALHĀO & 81 & CRIOULA BRANCA & 754 & MEXICANA-6 \\
\hline 33 & AMPELOPSIS WHITE & 82 & CUNNINGHAN & 755 & MISTURA \\
\hline 34 & AUGUST GIANT & 83 & D. MARIA & 756 & MOSCATEL BLUE \\
\hline 35 & B.1-151 & 84 & DATTIER DE BEIROUTH & 757 & MOSCATEL BRANCO ITALIA \\
\hline 36 & B.4-108 & 85 & DIAGALVES & 758 & MOSCATEL DE ALEXANDRIA \\
\hline 37 & B.31-164 & 86 & DIAMANTE NEGRO & 759 & MOSCATEL DE HAMBURGO \\
\hline 38 & B.40-208 & 87 & DIAMOND & 760 & MOSCATEL DE TERRACINA \\
\hline 39 & B.43-94 & 88 & DUTCHESS & 761 & MOSCATEL DONALISIO \\
\hline 40 & B.45-187 & 89 & EMERALD RIESLING & 762 & MOSCATEL DR. HOGG \\
\hline 41 & B.54-129 & 90 & EMPEROR & 763 & $\begin{array}{l}\text { MOSCATEL FLOR DE } \\
\text { LARANJEIRA }\end{array}$ \\
\hline 42 & B.66-168 & 91 & EMPIRE STATE & 764 & MOSCATEL ROSADA \\
\hline 43 & B.68-48 & 92 & EUMELAN & 765 & $\begin{array}{l}\text { MOSCATEL ROSSO DE } \\
\text { MALAGA }\end{array}$ \\
\hline 44 & BACO 22-A & 93 & F2-35 & 766 & NIAGARA BRANCA \\
\hline 45 & BAHIA & 94 & FERN MUNSON & 767 & NIAGARA DIPLÓIDE \\
\hline 46 & BARBERA & 95 & FLAME SEEDLESS & 768 & NIAGARA ROSADA \\
\hline 47 & BEAUTY SEEDLESS & 96 & FORNARINA & 769 & NIAGARA TETRAPLÓIDE \\
\hline 48 & BERTILLE SEYVE 460 & 97 & GOETHE & 770 & NOIR HATIF DE MARSEILLE \\
\hline 49 & BLACK JULY & 98 & GOLDEN MUSCAT & 771 & NORTON'S VIRGINIA \\
\hline
\end{tabular}

Sci. agric., Piracicaba, 52(1):107-122, jan./abr. 1995 
cont. TABELA 1

\begin{tabular}{|c|c|c|c|c|c|}
\hline 772 & NUGGET & 799 & S.R. $5012-3$ & 825 & SEIBEL 13.680 \\
\hline 773 & OLÍMPIA & 800 & S.S. & 826 & SEIBEL 14.583 \\
\hline 774 & P100-111 & 801 & SAN MARTINO & 827 & SÉMILLON \\
\hline 775 & PERLETTE & 802 & SAUVIGNON BLANC & 828 & SÉMILLON BRANCO \\
\hline 776 & PERLONA (PIROVANO 54) & 803 & SAUVIGNON GRIS & 829 & SOUZÃO \\
\hline 777 & PERLA DE CSABA & 804 & SAUVIGNON VERT & 830 & SULTANINA \\
\hline 778 & PIGNOLETTA & 805 & SEIBEL 2 & 831 & SYRAH \\
\hline 779 & PINOT CHARDONNAY & 806 & SEIBEL 1.077 & 832 & TAPACHULE \\
\hline 780 & PINOT TINTA & 807 & SEIBEL 5.213 & 833 & TRAVIÚ \\
\hline 781 & PIROVANO 24 & 808 & SEIBEL 5.455 & 834 & TREBBIANO \\
\hline 782 & PIROVANO 57 & 809 & SEIBEL 5.520 & 835 & Vitis caribaea \\
\hline 783 & PIZZUTELO BRANCO & 810 & SEIBEL 6.905 & 836 & Vitis cinerea \\
\hline 784 & PIZZUTELO NERO & 811 & SEIBEL 7.052 & 837 & Vitis gigas* \\
\hline 785 & PORTUGUESA AZUL & 812 & SEIBEL 7.053 & 838 & Vitis girdiana \\
\hline 786 & ROT GEWURZTRAMINER & 813 & SEIBEL 7.157 & 839 & Golia \\
\hline 787 & R.R. $101-14$ & 814 & SEIBEL 7.162 & 840 & Vitis lincecumii \\
\hline 788 & RED GLOBE & 815 & SEIBEL 7.222 & 841 & Vitis munsoniana \\
\hline 789 & RIESLING DO RHENO & 816 & SEIBEL 7.226 & 842 & Vitis riparia \\
\hline 790 & RIESLING SILVANER & 817 & SEIBEL 7.417 & 843 & Vitis roumdifolia \\
\hline 791 & ROSAKI ROSADO & 818 & SEIBEL 8.712 & 844 & V. mupestris/St. GEORGE \\
\hline 792 & ROSINHA & 819 & SEIBEL 8.745 & 845 & Vitis smalliana \\
\hline 793 & $\begin{array}{l}\text { MUSCAT ROUGE DE } \\
\text { MADERE }\end{array}$ & 820 & SEIBEL 10.076 & 846 & Vitis vinifera \\
\hline 794 & RUBI OKUYAMA & 821 & SEIBEL 10.096 & 847 & Wilkas \\
\hline 795 & RUBI CABERNET & 822 & SEIBEL 11.342 & 848 & X.Y.Z. \\
\hline 796 & RUBIRED & 823 & SEIBEL 12.583 & 849 & YORK MADEIRA \\
\hline 797 & V.shuttleworthii V.rufotomentosa & 824 & SEIBEL 13.053 & 850 & ZIBBIO DE PANTELARIA \\
\hline 798 & S.R. $496-15$ & & & \multicolumn{2}{|c|}{$(=$ MOSC DE ALEXANDRIA $)$} \\
\hline
\end{tabular}

* Provavelmente $V$.cinerea

A TABELA 1 traz a relação por ordem alfabética de todos os progenitores utilizados. Nos levantamentos efetuados até o momento, não se conseguiu identificar apropriadamente alguns deles, especificamente aqueles codificados apenas por números e alguns por número seguidos da letra $\mathrm{D}$.

Por outro lado, pode-se visualizar que as principais variedades da viticultura mundial foram utilizadas. Entretanto, das 850 relacionadas, 626 (73.6\%) possuiam a sigla IAC, identificando material oriundo do próprio programa, que receberam a numeração de 108 a 734, mas suprimidas da tabela por economia de espaço.

Catorze espécies de Vitis foram utilizadas com uma clara expectativa de ampliação da base genética dos variedades melhoradas, em busca de resistência a pragas e moléstias.
O uso de híbridos Seibel e Couderc foi amplo, mas poucos da série Bertille Seyve (apenas 1) e os da série Seyve Villard foram usados apenas nos JD.

Certamente pela dificuldade de obtenção, nenhum material proveniente de países do antigo Bloco Socialista foi usado, apesar da importância das variedades obtidas na Bulgária, Hungria, Yugoslávia e Rússia.

As duas variedades de uva de mesa mais plantadas em São Paulo, 'Italia' e 'Niagara Rosada' foram destacadamente as que mais vezes entraram nos cruzamentos. A explicação óbvia é que, se elas são as mais cultivadas é porque devem apresentar as caracteristicas mais apreciadas pelos consumidores e pelos produtores.

Em seguida, ainda com destaque, participando em mais de 100 cruzamentos, aparece 
'Patrícia', a melhor criação IAC e na verdade possuidora de grandes atributos, herdados principalmente de 'Moscatel de Hamburgo' e de 'Italia' que entram em sua genealogia.

Variedades de uvas com sabor moscatel estão entre as principais utilizadas, como 'Moscatel de Hamburgo', 'Moscatel Branco Italia' e 'Moscatel Rosada', certamente numa tentativa de mesclar essa característica com as de resistência, ou mesmo melhorar o sabor foxado de uvas americanas (SANTOS NETO, 1955).

'Soraya', participante de 68 cruzamentos, chegou a ser cultivada em São Paulo por suas boas qualidades, porém, um de seus defeitos, mais graves, a rachadura das bagas, tiroua de cultivo (POMMER, 1993). 'Soraya', entretanto, revelou-se excelente progenitor, como será discutido posteriormente.

'Cardinal' é a principal fonte de genes para precocidade o que, sem dúvida, é a razão para estar entre as variedades mais utilizadas nos cruzamentos.

Entre as IAC que participaram em mais de 20 cruzamentos, algumas não chegaram a receber nome de fantasia, não sendo reconhecidas propriamente como variedades. Entretanto, desempenharam importante papel como intermediárias na utilização de espécies silvestres no programa, como é o caso de IAC 339, IAC 392, IAC 393, IAC 394, IAC 405.

O número médio de sementes produzidas por cruzamento entre as variedades mais vezes utilizadas esteve entre 29 para Vitis caribaea $(=V$. tiliaefolia) e 163 para $V$. gigas (tudo indica que a espécie verdadeira é $V$. cinerea) duas espécies americanas silvestres. $O$ número é baixo, mas a literatura tem mostrado que muitas variedades foram selecionadas a partir de progênies de 15 a 30 plantas. Este fato demonstra a necessidade da boa escolha do progenitor e do correto direcionamento dos cruzamentos dentro do programa.

O estande final (médio) é bastante baixo, acentuando o que foi afirmado anteriormente, indo de 2 a 37 entre as variedades mais utilizadas. Do ponto de vista prático, estes dados, indicam a obrigatoriedade de se dedicar tratos culturais especiais às plantas que conseguem chegar à fase de campo. Aumentando a sobrevivência das plantas no campo, elevam-se as chances de selecionar melhor e de encontrar o indivíduo com as características desejadas.
Com este último aspecto em mente, foi calculado o "índice de sobrevivência", isto é, das plantas que chegaram ao campo, quantas conseguem se estabelecer de fato. Poucos cruzamentos conseguiram I.S. maior que $50 \%$.

As Figuras 1 e 2 ilustram as informações sobre as variedades mais utilizados como progenitores em todo o programa. $\mathrm{Na} 1^{\text {a }}$ estão as 5 primeiras e na segunda, as 5 seguintes, onde se pode notar, período por período o número de vezes em que cada uma foi utilizada. 'Italia', 'Niagara Rosada' e 'Patrícia' tiveram grande incre-mento de uso nos dois últimos períodos. Já 'Cardinal' teve o pico de utilização no período 4. 'Iracema' foi bastante utilizada no período 3 e no 6 .

As TABELAS 2 e 3 trazem as variedades utilizadas como progenitores femininos e progenitores masculinos, respectivamente. 'Italia', 'Niagara Rosada' e 'Patrícia' foram as mais utilizadas como progenitores femininos, em número de vezes bem maior que as outras variedades. As variedades estiveram também entre as mais utilizadas como progenitores masculinos.

Computando-se as variedades utilizadas em, no mínimo, 10 cruzamentos, algumas estiveram nos dois grupos: 'Dutchess', IAC 3393, 'Moscatel Rosada', 'Moscatel Branco Italia', IAC 392, 'Moscatel de Hamburgo', IAC 405-6, IAC 32-7, IAC 138-22, IAC 501-3/ Soraya, IAC 871-41/Patrícia, 'Niagara Rosada' e 'Italia'. Este fato demonstra que, além destas variedades apresentarem flores perfeitas, devem somar bons atributos para ter sido escolhidas.

Diversas espécies silvestres foram usadas como progenitores femininos, mercê de sua capacidade de produzir frutos $\mathrm{e}$ sementes, especialmente $V$. gigas e $V$. shutlleworthii $\times V$. rufotomentosa. Já $V$. caribaea, $V$. smalliana, $V$. cinerea e $V$. rotundifolix foram utilizadas mais intensamente como polinizadores, devido à sua baixa capacidade de produzir frutos (ou incapacidade total). No caso de $V$. rotundifolia é preciso destacar a diferença no número de cromossomos, o que torna inférteis os poucos hîbridos que eventualmente são obtidos.

As Figuras 3 a 6 mostram a utilização, por período, das variedades como progenitores femininos e masculinos. 'Italia' e 'Niagara Rosada' foram intensamente usadas no período 6 como mães. Por outro lado, 'Italia' foi mais usada como pai no período 2 . 
TABELA 2 - Variedades de videira utilizadas como progenitores femininos no programa de melhoramento da videira no IAC, de 1943 a 1990 , com seus respectivos números de identificação (N), número total de vezes que foi utilizada (T), estande inicial (Ei) e final (Ef) médios das progênies obtidas dos cruzamentos, índice de sobrevivência médio (IS $=100 * E f / E i) ~ e$ porcentagem final de plantas no campo em relação ao número de sementes produzidas.

\begin{tabular}{|c|c|c|c|c|c|c|}
\hline $\mathrm{N}$ & VARIEDADE & $\mathrm{T}$ & $\mathrm{Ei}$ & Ef & IS & $\mathbf{P}$ \\
\hline 736 & ITALIA & 141 & 2310 & 885 & 38 & 9 \\
\hline 768 & NIAGARA ROSADA & 141 & 6199 & 3326 & 54 & 17 \\
\hline 448 & IAC 871-41/PATRÍCIA & 95 & 751 & 102 & 14 & 3 \\
\hline 824 & SEIBEL 13.053 & 36 & 705 & 292 & 41 & 16 \\
\hline 253 & IAC 501-6/SORAYA & 27 & 548 & 163 & 30 & 9 \\
\hline 822 & SEIBEL 11.342 & 25 & 300 & 79 & 26 & 4 \\
\hline 167 & IAC 138-22/MÁXIMO & 24 & 721 & 148 & 21 & 4 \\
\hline 223 & IAC 394-13 & 24 & 352 & 204 & 58 & 16 \\
\hline 224 & IAC 394-14 & 23 & 363 & 240 & 66 & 19 \\
\hline 141 & IAC $32-7$ & 21 & 574 & 201 & 35 & 10 \\
\hline 812 & SEIBEL 7.053 & 20 & 731 & 135 & 18 & 9 \\
\hline 146 & IAC $65-A 2$ & 19 & 485 & 216 & 45 & 12 \\
\hline 485 & IAC 931-13/TETÊ & 19 & 174 & 63 & 36 & 3 \\
\hline 233 & IAC $405-6$ & 18 & 657 & 268 & 41 & 14 \\
\hline 739 & JD 930 & 18 & 101 & $\mathbf{0}$ & 0 & 0 \\
\hline 837 & Vitis gigas & 18 & 931 & 216 & 23 & 6 \\
\hline 98 & GOLDEN MUSCAT & 17 & 120 & 71 & 59 & 9 \\
\hline 106 & HIGHLAND & 16 & 253 & 43 & 17 & 4 \\
\hline 150 & IAC $74-4 /$ IARA & 16 & 122 & 61 & 50 & 5 \\
\hline 759 & MOSCATEL DE HAMBURGO & 16 & 194 & 65 & 34 & 8 \\
\hline 218 & IAC $393-5$ & 15 & 239 & 28 & 12 & 4 \\
\hline 826 & SEIBEL 14.583 & 15 & 210 & 81 & 39 & 8 \\
\hline 216 & IAC 392 & 14 & 701 & 204 & 29 & 14 \\
\hline 296 & IAC 554-8 & 14 & 165 & 66 & 40 & 8 \\
\hline 439 & IAC $861-3$ & 14 & 195 & 106 & 54 & 13 \\
\hline 757 & MOSCATEL BRANCO ITALIA & 14 & 205 & 57 & 28 & 8 \\
\hline 764 & MOSCATEL ROSADA & 14 & 256 & 93 & 36 & 9 \\
\hline 80 & COUDERC 17 & 13 & 122 & 31 & 25 & 4 \\
\hline 198 & IAC 339-3 & 13 & 104 & 89 & 86 & 27 \\
\hline 273 & IAC 526-4 & 13 & 271 & 206 & 76 & 30 \\
\hline 797 & V.shuttleworthii V.rufotomentosa & 13 & 322 & 136 & 42 & 10 \\
\hline 814 & SEIBEL 7.162 & 13 & 61 & 19 & 31 & 6 \\
\hline 88 & DUTCHESS & 12 & 17 & 3 & 18 & 2 \\
\hline 156 & IAC 82-1 & 12 & 225 & 83 & 37 & 19 \\
\hline 225 & IAC 394-16 & 12 & 608 & 118 & 19 & 11 \\
\hline 697 & IAC 1615 & 12 & 260 & 138 & 53 & 19 \\
\hline 2 & 62-D10 & 11 & 389 & 188 & 48 & 13 \\
\hline 4 & 432-D10 & 11 & 170 & 39 & 23 & 8 \\
\hline 6 & 493-D10 & 11 & 46 & 12 & 26 & 2 \\
\hline 699 & IAC $1616-2$ & 11 & 343 & 195 & 57 & 16 \\
\hline 766 & NIAGARA BRANCA & 11 & 305 & 243 & 80 & 22 \\
\hline
\end{tabular}

Sci. agric., Piracicaba, 52(1):107-122, jan./abr. 1995 
TABELA 3. Variedades de videira utilizadas como progenitores masculinos, no programa de melhoramento do IAC, de 1943 a 1990 , com seus respectivos números de identificação (N), número total de vezes que foi utilizada (T), estande inicial (Ei) e final (Ef) médios das progếnies obtidas dos cruzamentos, índice de sobrevivência médio ( $\mathrm{IS}=100 * \mathrm{Ef} / \mathrm{Ei}$ ) e porcentagem final de plantas no campo em relação ao número de sementes produzidas (P).

\begin{tabular}{|c|c|c|c|c|c|c|}
\hline $\mathbf{N}$ & VARIEDADE & $\mathbf{T}$ & $\mathrm{Ei}$ & Ef & IS & $\mathbf{P}$ \\
\hline 759 & MOSCATEL DE HAMBURGO & 62 & 1134 & 349 & 31 & 6 \\
\hline 736 & TALIA & 42 & 938 & 245 & 26 & 5 \\
\hline 67 & CARDINAL & 41 & 1636 & 400 & 24 & 9 \\
\hline 253 & IAC 501-6/SORAYA & 40 & 454 & 289 & 64 & 11 \\
\hline 757 & MOSCATEL BRANCO ITALIA & 38 & 511 & 159 & 31 & 6 \\
\hline 764 & MOSCATEL ROSADA & 34 & 314 & 59 & 19 & 4 \\
\hline 245 & IAC 457-11/IRACEMA & 32 & 990 & 491 & 50 & 16 \\
\hline 88 & DUTCHESS & 30 & 551 & 89 & 16 & 6 \\
\hline 786 & ROT GEWÜRZTRAMINER & 28 & 377 & 150 & 40 & 9 \\
\hline 746 & MADRESFIELD COURT & 27 & 626 & 214 & 34 & 9 \\
\hline 831 & SYRAH & 27 & 433 & 181 & 42 & 9 \\
\hline 751 & MERLOT & 26 & 436 & 182 & 42 & 5 \\
\hline 100 & GOLDEN QUEEN & 24 & 416 & 110 & 26 & 6 \\
\hline 183 & IAC $287-2$ & 23 & 399 & 133 & 33 & 10 \\
\hline 167 & IAC 138-22/MÁXIMO & 22 & 317 & 181 & 57 & 10 \\
\hline 246 & IAC 460-1/HELENA & 22 & 646 & 273 & 42 & 12 \\
\hline 768 & NIAGARA ROSADA & 21 & 569 & 71 & 12 & 2 \\
\hline 287 & IAC 544-14 & 20 & 833 & 390 & 47 & 15 \\
\hline 795 & RUBI CABERNET & 20 & 436 & 184 & 42 & 16 \\
\hline 780 & PINOT TINTA & 19 & 166 & 55 & 33 & 4 \\
\hline 835 & Vitis caribaea & 19 & 123 & 32 & 26 & 5 \\
\hline 137 & IAC 21-14/MADALENA & 18 & 419 & 76 & 18 & 5 \\
\hline 284 & IAC 540-3 & 18 & 497 & 349 & 70 & 16 \\
\hline 66 & CABERNET SAUVIGNON & 17 & 376 & 142 & 38 & 8 \\
\hline 205 & IAC 344-2/ANGÉLICA & 17 & 262 & 77 & 29 & 8 \\
\hline 217 & IAC 393-4 & 17 & 267 & 90 & 34 & 11 \\
\hline 377 & IAC 768-2 & 17 & 529 & 222 & 42 & 14 \\
\hline 741 & JUMBO & 17 & 1000 & 382 & 38 & 21 \\
\hline 770 & NOIR HÂTIF DE MARSEILLE & 17 & 547 & 256 & 47 & 13 \\
\hline 830 & SULTANINA & 17 & 220 & 52 & 24 & 5 \\
\hline 30 & ALIGOTÉ & 16 & 465 & 117 & 25 & 8 \\
\hline 779 & PINOT CHARDONNAY & 16 & 285 & 157 & 55 & 13 \\
\hline 827 & SEMILLON & 16 & 191 & 80 & 42 & 5 \\
\hline 845 & Vitis smalliana & 16 & 390 & 199 & 51 & 14 \\
\hline 233 & IAC $405-6$ & 15 & 84 & 35 & 42 & 10 \\
\hline 382 & IAC 775-26/AURORA & 15 & 935 & 804 & 86 & 33 \\
\hline 446 & IAC 871-13/A DONA & 15 & 1068 & 616 & 58 & 21 \\
\hline 448 & IAC 871-41/PATRfCIA & 15 & 555 & 7 & 1 & 0 \\
\hline 758 & MOSCATEL DE ALEXANDRIA & 15 & 219 & 92 & 42 & 11 \\
\hline 775 & PERLETTE & 15 & 595 & 272 & 46 & 12 \\
\hline 777 & PERLA DE CSABA & 15 & 292 & 65 & 22 & 6 \\
\hline 836 & Vitis cinerea & 15 & 154 & 92 & 60 & 4 \\
\hline 216 & LAC 392 & 14 & 135 & 73 & 54 & 11 \\
\hline
\end{tabular}

Sci. agric., Piracicaba, 52(1):107-122, jan./abr. 1995 
cont. TABELA 3

\begin{tabular}{lllllll}
\hline 265 & IAC 514-6/MARIA & 13 & 860 & 645 & 75 & 35 \\
45 & BAHIA & 12 & 144 & 34 & 24 & 6 \\
174 & IAC 181 & 12 & 361 & 87 & 24 & 9 \\
247 & IAC 470-26 & 12 & 352 & 148 & 42 & 14 \\
791 & ROSAKI ROSADO & 12 & 119 & 34 & 29 & 8 \\
843 & Vitis rotundifolia & 12 & 58 & 17 & 29 & 7 \\
53 & BURGUNDER KATENHOLZ & 11 & 402 & 97 & 24 & 11 \\
283 & IAC 536-2/MORENINHA & 11 & 414 & 94 & 23 & 6 \\
473 & IAC 901-1 & 11 & 744 & 129 & 17 & 6 \\
474 & IAC 903-15 & 11 & 315 & 56 & 18 & 6 \\
769 & NIAGARA TETRAPLÓIDE & 11 & 52 & 0 & 0 & 0 \\
803 & SAUVIGNON GRIS & 11 & 159 & 115 & 72 & 12 \\
\hline \hline
\end{tabular}

'Cardinal' foi praticamente utilizada como progenitor masculino e intensamente no período 4. 'Dutchess' e 'Moscatel Rosada' foram usados como fontes de pólen no período 1 , principalmente.

'Seibel 11.342', 'Seibel 13.053' foram utilizadas apenas no período 1 e como mães, provavelmente substituídas por híbridos mais recentes.

É fácil observar que somente na TABELA 3 encontram-se variedades apirenas (sem sementes) como 'Iracema', 'Sultanina', IAC 54414, IAC 287-2, IAC 540-3, 'Jumbo', 'Aurora', 'A Dona', 'Perlette', 'Maria' e outros, especialmente entre as mais usadas. Até o final dos anos 80 a obtenção de nova variedade apirena só era possível mediante a passagem por progenitor que apresentasse sementes, polinizada por pólen de variedade apirena. Com a utilização da técnica de resgate de embrióes, (PASSOS et al., 1992) tornou-se possível o cruzamento direto entre duas variedades apirenas. Em 1989 foram feitos os primeiros cruzamentos dessa natureza no IAC, juntamente com a tendência mundial.

Quanto à finalidade dos cruzamentos, a Figura 7 mostra a proporção de utilização de progenitores para obtenção de uvas apirenas. Percebe-se, entre as dez variedades mais utilizadas, uma distribuição semelhante entre apirenas e com sementes.

Sci. agric., Piracicaba, 52(1):107-122, jan./abr. 1995
Na Figura 8, para uvas de mesa em geral, a grande predominância , 'Cardinal', 'Italia' e 'Soraya'. Neste caso, as dez variedades mais utilizadas entraram em $44,7 \%$ dos cruzamentos com essa finalidade, comparando-se com os $26,4 \%$ da Figura 1.

A Figura 9 exibe a proporção das variedades mais utilizadas para uvas de vinho. É interessante notar que as dez mais usadas o foram em porcentagens próximas de $10 \%$ (de 9,0 a $12,5 \%$ ) sendo 6 viníferas puras e 4 híbridos.

$\mathrm{Na}$ TABELA 4 estão relacionadas as variedades submetidas à autofecundação. Foram apenas $52 \mathrm{em} 48$ anos de programa, decididamente um número muito pequeno. É provável que, à semelhança de programas de melhoramento de outras plantas, tenha havido um certo receio de incrementar a endogamia. Entretanto, examinando-se a genealogia das variedades IAC (Anexo I) verificase que muitos deles apresentam uma complexidade enorme. Este fato daria suficiente embasamento teórico para se tentar um número muito maior de autofecundações tentando explorar ao máximo a agregação conseguida através dos anos.

A TABELA 5 traz a relação das variedades que apresentaram porcentagem de plantas no campo, em relação ao número de sementes, maior que $30 \%$. Observa-se que o maior valor encontrado foi de $80 \%$, para um número médio de sementes (5) muito baixo. 


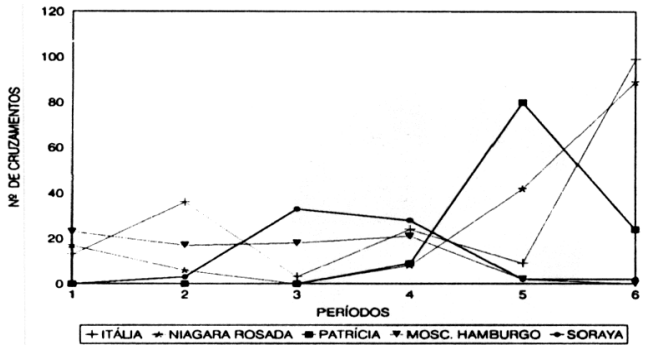

Figura 1 - Número total de vezes que a variedade foi utilizada como progenitor feminino, masculino e autofecundada por período (5 primeiros).

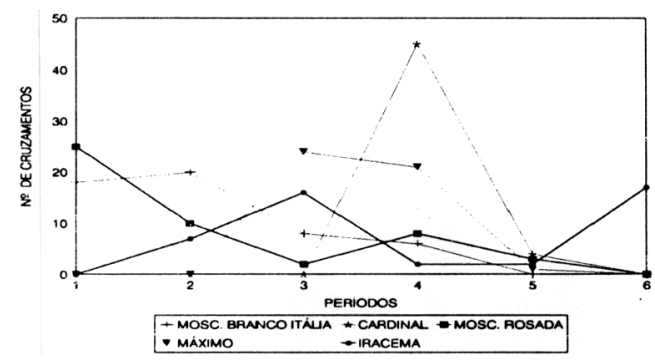

Figura 2 - Número total de vezes que a variedade foi utilizada como progenitor feminino, masculino e autofecundada por periodo (5 primeiros).

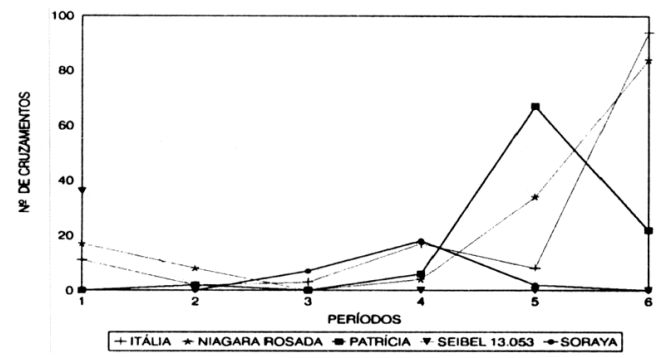

Figura 3 - Número total de vezes que a variedade foi utilizada como progenitor feminino, por período (5 primeiros).

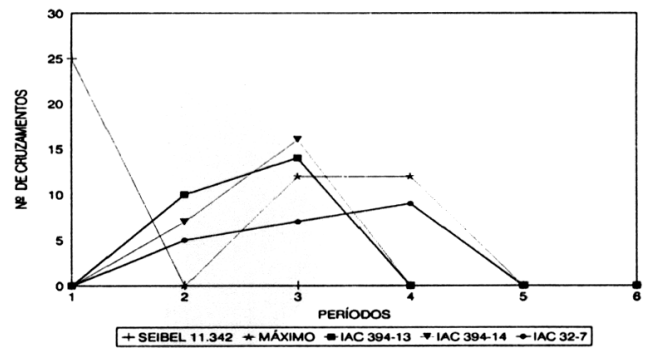

Figura 4 - Número total de vezes que a variedade foi utilizada como progenitor feminino, por período ( 5 primeiros).

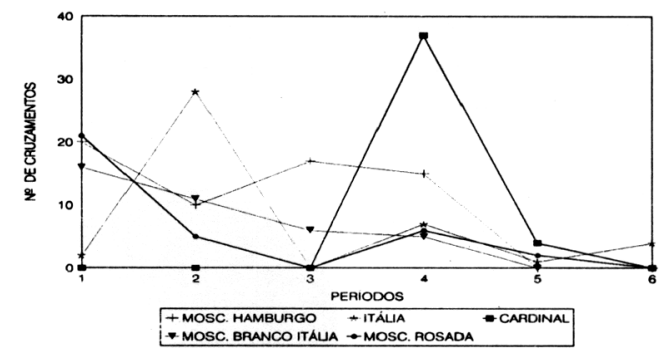

Figura 5 - Número total de vezes que a variedade foi utilizada como progenitor masculino, por período (5 primeiros).

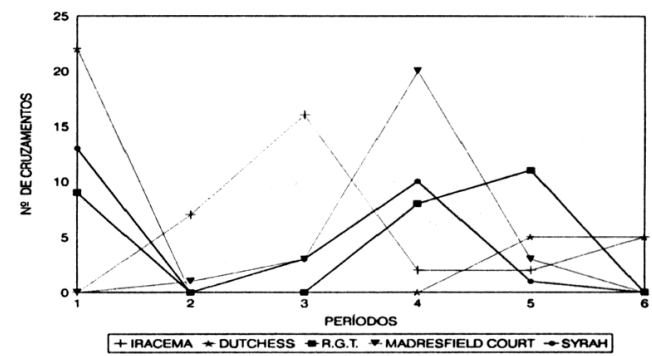

Figura 6 - Número total de vezes que a variedade foi utilizada como progenitor masculino, por período (5 primeiros).

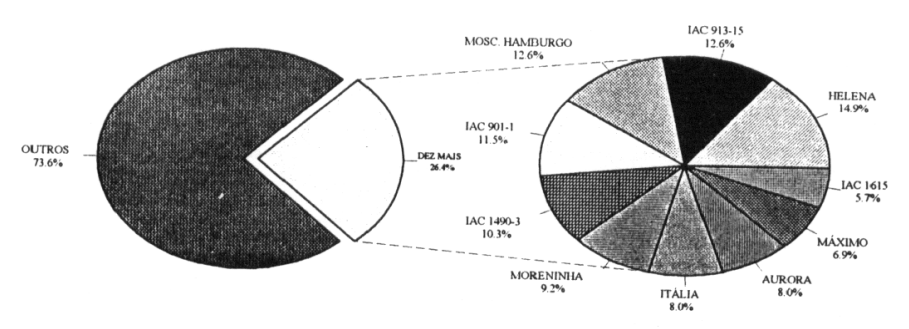

A

B

Figura 7 - Número total de variedades utilizadas em cruzamentos para obtenção de uvas apirenas. A) As dez variedades mais utilizadas em relação ao número total. B) Proporção de uso das dez variedades mais utilizadas. 


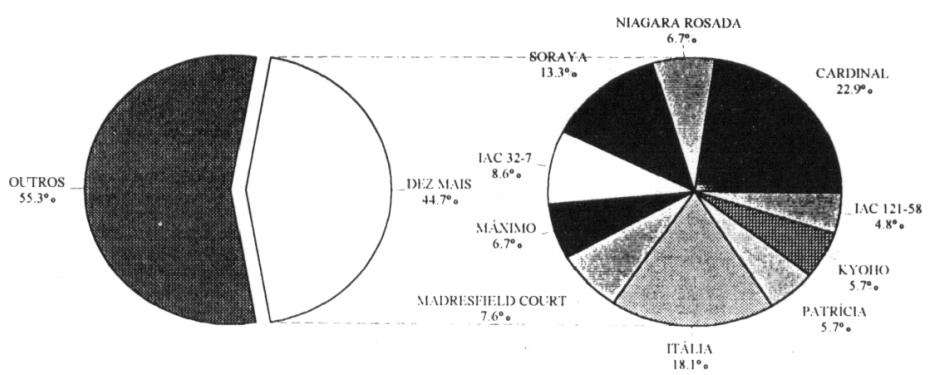

A

B

Figura 8 - Número total de variedades utilizadas em cruzamentos para obtenção de uva de mesa. A) As dez variedades mais utilizadas em relação ao número total. B) Proporção de uso das dez variedades mais utilizadas.

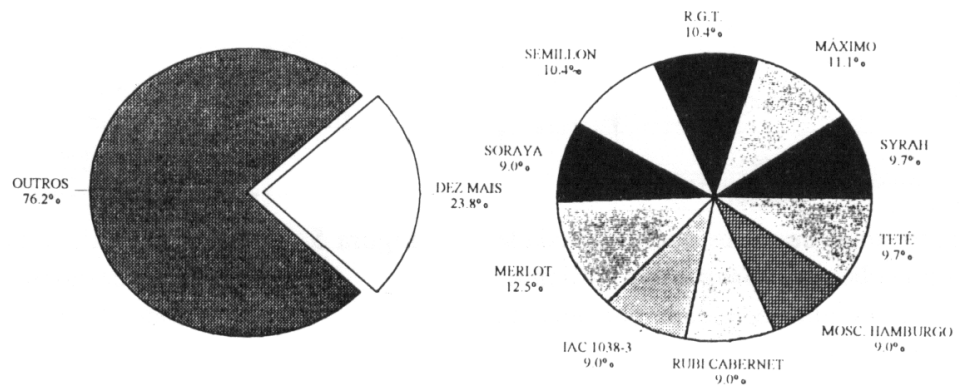

A

B

Figura 9 - Número total de variedades utilizadas em cruzamentos para obtenção de uvas para vinho. A) As dez variedades mais utilizadas em relação ao número total. B) Proporção de uso das dez variedades mais utilizadas.

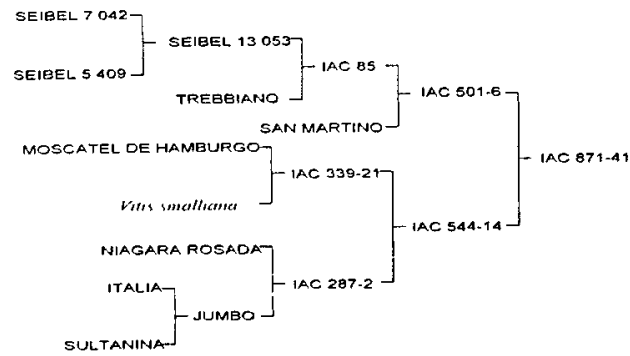

Figura 10 - Genealogia da variedade IAC-871-41/ PATRÍCIA

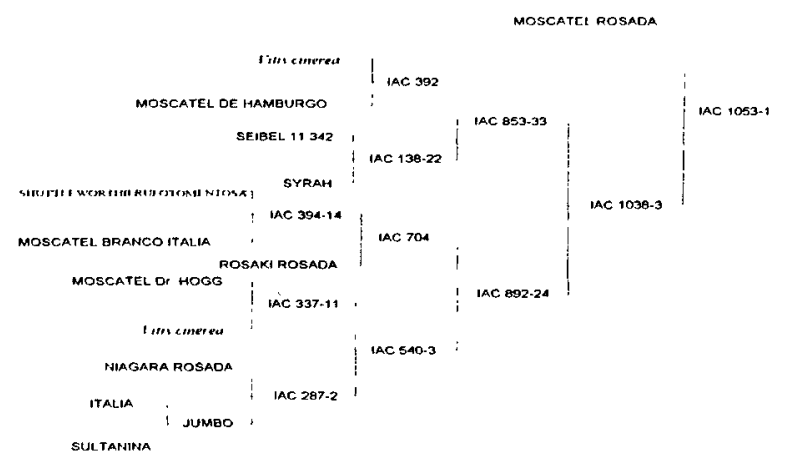

Figura 11 - Genealogia da variedade IAC-13531/YOLE

Sci. agric., Piracicaba, 52(1):107-122,jan./abr. 1995 


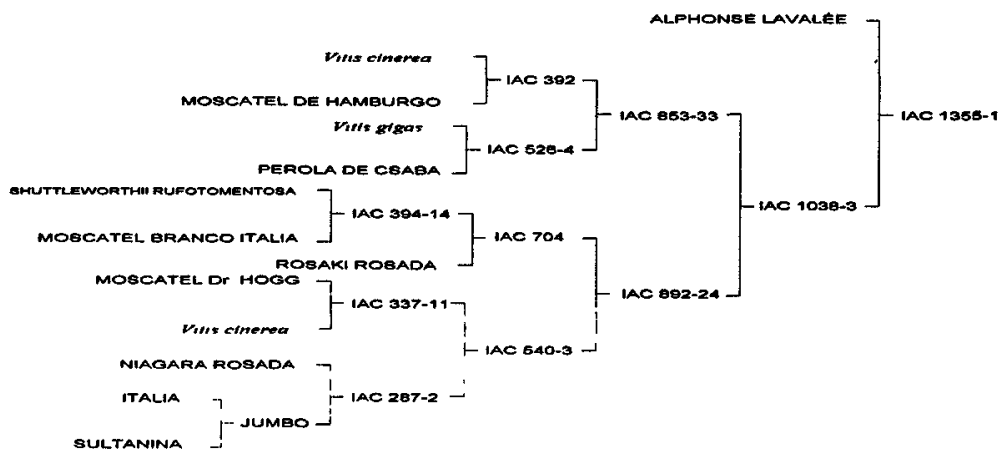

Figura 12 - Genealogia da variedade IAC-1355-1/GRÃO MOGOL

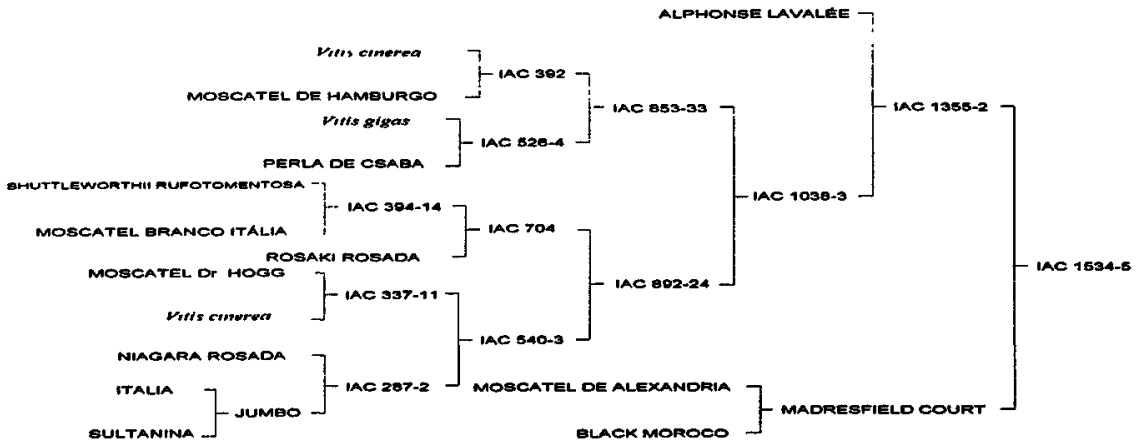

Figura 13 - Genealogia da variedade IAC-1534-5/MARILIA

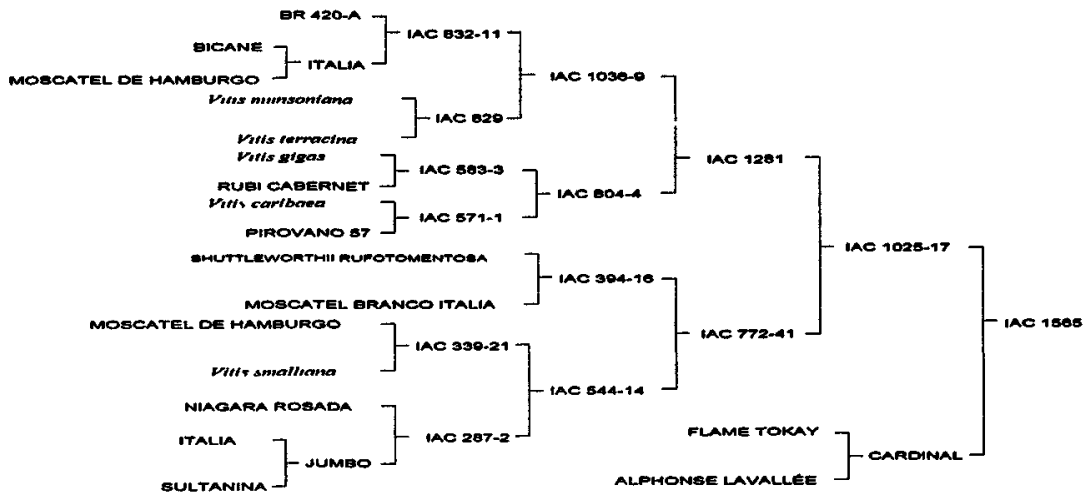

Figura 14 - Genealogia da variedade IAC-1562/CAROLINA

Sci. agric., Piracicaba, 52(1):107-122, jan./abr. 1995 
TABELA 4 - Variedades de videira, autofecundadas. N-número de identificação; T-total de vezes que foi utilizada; Ei e Ef-estande inicial e final médios; IS-índice de sobrevivência médio e Pporcentagem final de plantas no campo.

\begin{tabular}{|c|c|c|c|c|c|c|}
\hline VARIEDADE & $\mathbf{N}$ & $\mathbf{T}$ & $\mathrm{Ei}$ & Ef & IS & $\mathbf{P}$ \\
\hline IAC 871-41/PATRÍCIA & 448 & 3 & 251 & 104 & 41 & 13 \\
\hline 4416-2 & 10 & 2 & 34 & 10 & 29 & 7 \\
\hline 432-D10 & 4 & 1 & 15 & 4 & 27 & 4 \\
\hline $4421-4$ & 12 & 1 & 1 & 1 & 100 & 1 \\
\hline $4421-10$ & 13 & 1 & 6 & 2 & 33 & 4 \\
\hline $4423-2$ & 14 & 1 & 0 & 0 & 0 & 0 \\
\hline 46164-20 & 19 & 1 & 12 & 8 & 67 & 11 \\
\hline CHILE NORMANHA MOSCATO & 76 & 1 & 0 & $\mathbf{0}$ & 0 & 0 \\
\hline GOETHE & 97 & 1 & 0 & 0 & 0 & $\mathbf{0}$ \\
\hline IAC 16-2 & 132 & 1 & 0 & $\mathbf{0}$ & 0 & 0 \\
\hline IAC $121-58$ & 162 & 1 & 27 & 1 & 4 & 0 \\
\hline IAC 181 & 174 & 1 & 8 & 1 & 12 & 1 \\
\hline IAC 313/TROPICAL & 184 & 1 & 13 & 0 & 0 & 0 \\
\hline IAC 337-11 & 194 & 1 & 32 & 32 & 100 & 40 \\
\hline IAC 338-4 & 195 & 1 & 22 & 0 & 0 & 0 \\
\hline IAC 338-27 & 197 & 1 & 18 & 0 & 0 & 0 \\
\hline IAC 339-3 & 198 & 1 & 11 & 11 & 100 & 10 \\
\hline IAC 339-19 & 201 & 1 & 17 & 17 & 100 & 10 \\
\hline IAC 392 & 216 & 1 & 6 & 6 & 100 & 14 \\
\hline IAC 393-4 & 217 & 1 & 25 & 0 & 0 & 0 \\
\hline IAC 501-6/SORAYA & 253 & 1 & 56 & 8 & 14 & 5 \\
\hline IAC 503-33/MARIETA & 259 & 1 & 36 & 2 & 6 & 1 \\
\hline IAC $565-2$ & 299 & 1 & 11 & 1 & 9 & 2 \\
\hline IAC 565-3 & 300 & 1 & 2 & 1 & 50 & 6 \\
\hline IAC 571 -1 & 306 & 1 & 25 & 0 & 0 & 0 \\
\hline IAC 581-7 & 316 & 1 & 5 & 1 & 20 & 4 \\
\hline IAC 583-3 & 318 & 1 & 1 & $\mathbf{0}$ & 0 & 0 \\
\hline IAC 589-2 & 323 & 1 & 12 & 0 & 0 & 0 \\
\hline IAC $821-44$ & 409 & 1 & 33 & 16 & 48 & 16 \\
\hline IAC 829 & 421 & 1 & 26 & 20 & 77 & 15 \\
\hline IAC 842-4/EUGÊNIO & 423 & 1 & 0 & 0 & 0 & $\mathbf{0}$ \\
\hline IAC 931-13/TETE & 485 & 1 & 383 & 47 & 12 & 8 \\
\hline IAC 989 & 507 & 1 & 2 & 2 & 100 & 1 \\
\hline IAC 1038-3 & 538 & 1 & 0 & 0 & $\mathbf{0}$ & $\mathbf{0}$ \\
\hline IAC $1430-10$ & 661 & 1 & 0 & 0 & 0 & 0 \\
\hline IAC $1430-11$ & 662 & 1 & 17 & 2 & 12 & 2 \\
\hline IAC $1675-2$ & 707 & 1 & 0 & 0 & 0 & 0 \\
\hline
\end{tabular}

Sci. agric., Piracicaba, 52(1):107-122, jan./abr. 1995 


\begin{tabular}{lllllll}
\hline IAC 1726-7/MARIA ROSA & 712 & 1 & 0 & 0 & 0 & 0 \\
IAC 1792 & 723 & 1 & 18 & 17 & 94 & 22 \\
IAC $1848-7$ & 726 & 1 & 14 & 0 & 0 & 0 \\
IAC $1849-1$ & 727 & 1 & 13 & 0 & 0 & 0 \\
IAC $1897-6$ & 730 & 1 & 16 & 0 & 0 & 0 \\
IAC $1897-16$ & 731 & 1 & 14 & 0 & 0 & 0 \\
IAC $1897-18$ & 732 & 1 & 0 & 0 & 0 & 0 \\
IAC $1899-6$ & 733 & 1 & 0 & 0 & 0 & 0 \\
ITALIA & 736 & 1 & 31 & 8 & 26 & 12 \\
JD 874 & 738 & 1 & 0 & 0 & 0 & 0 \\
S.R. 496-15 & 800 & 1 & 21 & 17 & 81 & 14 \\
TAPACHULE & 834 & 1 & 2 & 2 & 100 & 100 \\
\hline \hline
\end{tabular}

TABELA 5 - Variedades de videira utilizadas no programa de melhoramento do IAC, de 1943 a 1990, com seus respectivos números de identificação $(\mathrm{N})$, número total de vezes que foi utilizada $(\mathrm{T})$, número médio de sementes produzidas (S), estande inicial (Ei) e final (Ef) médios, índice de sobrevivência médio (IS) e porcentagem final de plantas no campo em relação ao número de sementes produzidas $(\mathrm{P})$, para $\mathrm{P}>30 \%$.

\begin{tabular}{lllccccl}
\hline \hline $\mathbf{N}$ & VARIEDADE & $\mathrm{T}$ & $\mathrm{S}$ & $\mathrm{Ei}$ & $\mathrm{Ef}$ & $\mathrm{IS}$ & $\mathrm{P}$ \\
\hline 354 & IAC 706 & $\mathbf{I}$ & 5 & 5 & 4 & 80 & 80 \\
395 & IAC 804-4 & 2 & 84 & 69 & 60 & 87 & 71 \\
709 & IAC 1680-5 & 1 & 91 & 62 & 44 & 71 & 48 \\
201 & IAC 339-19 & 2 & 94 & 63 & 64 & 100 & 67 \\
16 & $4436-9$ & 1 & 6 & 4 & 4 & 100 & 67 \\
140 & IAC 32-6 & 5 & 5 & 3 & 3 & 100 & 60 \\
422 & IAC 832-11 & 2 & 83 & 57 & 51 & 89 & 55 \\
553 & IAC 1088-6 & 1 & 2 & 1 & 1 & 100 & 50 \\
410 & IAC 822-9 & 2 & 28 & 21 & 15 & 71 & 53 \\
196 & IAC 338-6 & 1 & 20 & 10 & 8 & 80 & 40 \\
371 & IAC 738-2 & 1 & 70 & 35 & 28 & 80 & 40 \\
192 & IAC 337-9 & 1 & 23 & 9 & 9 & 100 & 39 \\
753 & MEXICANA-3 & 1 & 89 & 39 & 35 & 90 & 39 \\
197 & IAC 338-24 & 1 & 13 & 5 & 5 & 100 & 38 \\
268 & IAC 520-1 & 1 & 38 & 19 & 14 & 74 & 36 \\
367 & IAC 721-1 & 2 & 16 & 11 & 8 & 73 & 36 \\
472 & IAC 892-24 & 2 & 59 & 27 & 20 & 74 & 34 \\
344 & IAC 670 & 1 & 3 & 2 & 1 & 50 & 33 \\
417 & IAC 823-46 & 1 & 12 & 6 & 4 & 67 & 33 \\
430 & IAC 849-23 & 1 & 18 & 9 & 6 & 67 & 33 \\
681 & IAC 1532-9 & 1 & 30 & 12 & 10 & 83 & 33 \\
552 & IAC 1087-5 & 1 & 62 & 31 & 19 & 61 & 31 \\
\hline \hline
\end{tabular}


TABELA 6 - Variedades de videira utilizadas no programa de melhoramento do IAC, de 1943 a 1990 , com seus respectivos números de identificação $(\mathrm{N})$, número total de vezes que foi utilizada $(T)$, número médio de sementes produzidas (S), estande inicial (Ei) e final (Ef) médios, índice de sobrevivência médio (IS) e porcentagem final de plantas no campo em relação ao número de sementes produzidas (P), para $S>200$ sementes.

\begin{tabular}{|c|c|c|c|c|c|c|c|}
\hline $\mathbf{N}$ & VARIEDADE & $\mathrm{T}$ & s & $\mathrm{Ei}$ & Ef & IS & $\mathbf{P}$ \\
\hline 524 & IAC $1009-35$ & 1 & 661 & 7 & 4 & 57 & 1 \\
\hline 525 & IAC 1009-77 & 1 & 587 & 388 & 11 & 3 & 2 \\
\hline 583 & IAC $1164-1$ & 1 & 573 & 39 & 37 & 95 & 6 \\
\hline 768 & NIAGARA DIPLÓIDE & 1 & 504 & 16 & 0 & 0 & 0 \\
\hline 596 & IAC 1228-1 & 1 & 454 & 128 & 70 & 55 & 15 \\
\hline 770 & NIAGARA TETRAPLÓIDE & 1 & 446 & 4 & 0 & 0 & 0 \\
\hline 586 & IAC $1177-6$ & 2 & 409 & 34 & 20 & 59 & 5 \\
\hline 738 & JD 874 & 1 & 402 & 162 & 36 & 22 & 8 \\
\hline 539 & IAC 1038-4 & 1 & 377 & 13 & 7 & 54 & 2 \\
\hline 497 & IAC $962-8$ & 1 & 366 & 18 & 6 & 33 & 2 \\
\hline 665 & IAC $1441-13$ & 1 & 365 & 217 & 62 & 29 & 17 \\
\hline 522 & IAC $1009-27$ & 1 & 335 & 110 & 20 & 18 & 6 \\
\hline 21 & A. $8-158$ & 2 & 328 & 4 & 0 & 0 & 0 \\
\hline 511 & IAC 994-7 & 2 & 320 & 65 & 26 & 40 & 8 \\
\hline 153 & IAC 74-7 & 3 & 317 & 36 & 10 & 28 & 3 \\
\hline 426 & IAC $846-4$ & 1 & 303 & 26 & 10 & 38 & 3 \\
\hline 9 & $4313-12$ & 2 & 296 & 62 & 24 & 39 & 8 \\
\hline 513 & IAC $997-21$ & 1 & 284 & 27 & 16 & 59 & 6 \\
\hline 651 & IAC $1399-11$ & 1 & 266 & 40 & 28 & 70 & 11 \\
\hline 569 & IAC 1122-1 & 1 & 258 & 20 & 14 & 70 & 5 \\
\hline 518 & IAC $1009-12$ & 3 & 251 & 23 & 14 & 61 & 5 \\
\hline 571 & IAC 1131 & 1 & 251 & 15 & 13 & 87 & 5 \\
\hline 534 & IAC $1035-12$ & 1 & 250 & 25 & 24 & 96 & 10 \\
\hline 587 & IAC 1179 & 1 & 245 & 28 & 2 & 7 & 1 \\
\hline 491 & IAC $960-6$ & 6 & 244 & 52 & 11 & 21 & 5 \\
\hline 819 & SEIBEL 8.712 & 2 & 239 & 34 & 26 & 76 & 11 \\
\hline 544 & IAC $1062-14$ & 1 & 226 & 4 & 2 & 50 & 1 \\
\hline 451 & IAC $875-10$ & 1 & 221 & 13 & 9 & 69 & 4 \\
\hline 372 & IAC $746-3$ & 3 & 220 & 86 & 12 & 14 & 5 \\
\hline 494 & IAC $960-12$ & 1 & 217 & 76 & 30 & 39 & 14 \\
\hline 500 & IAC $966-9$ & 6 & 216 & 50 & 1 & 20 & 1 \\
\hline 837 & Vitis gigas & 18 & 215 & 52 & 12 & 23 & 6 \\
\hline 414 & IAC $823-24$ & 2 & 214 & 30 & 17 & 57 & 8 \\
\hline 556 & IAC $1100-12$ & 1 & 214 & 69 & 30 & 43 & 14 \\
\hline 493 & IAC 960-11 & 1 & 212 & 4 & 2 & 50 & 1 \\
\hline 447 & IAC $871-18$ & 4 & 205 & 55 & 18 & 33 & 9 \\
\hline
\end{tabular}

Sci. agric., Piracicaba, 52(1):107-122, jan./abr. 1995 
Nenhuma das famílias das variedades relacionadas na TABELA 5 tiveram plantas selecionadas diretamente, mas 11 variedades surgiram de famílias com mais de 3 plantas e das famílias mais numerosas. Outro feito digno de nota é que, excetuando-se 4436-9 (desconhecido) e IAC 32-6, as outras variedades com IS $=100 \%$ foram resultantes de cruzamentos com $V$. cinerea diretamente (IAC 337, 338, 339) ou de autofecun-dações de um destes (IAC 1088 = IAC 338-4). Certamente, a espécie silvestre conferiu resistência de campo a essas variedades, aumentando sua sobrevivência.

Os que apresentaram maior número de sementes entre esses 22 , possuem como ancestral pelo menos uma espécie silvestre, quase sempre $V$. cinerea e algumas vezes, $V$. caribaea.

$\mathrm{Na}$ TABELA 6 foram relacionadas as variedades cujos cruzamentos produziram, no mínimo, 200 sementes. Pode-se observar que, das 36 variedades, apenas 5 apresentaram estande inicial abaixo de 10 plantas, incluindo-se aí a Niagara tetraplóide, o que seria de se esperar, a Niagara chamada de diplóide mas, certamente, alguma mutação também a nível cromossômico, e o A8-158 (desconhecido).

Apesar do alto número de sementes, o estande inicial foi maior que 100 plantas em apenas 5 casos. O estande inicial elevado também não foi garantia de grande número de plantas no campo, pois desses 5 casos, apenas dois mostraram $P$ maior que $10 \%$. O estande final foi razoável, em termos de videira, pois a maioria das variedades relacionados teve mais de 10 plantas no campo e 13 tiveram 20 ou mais plantas, melhorando a chance de sucesso na identificação de algum tipo superior.

Deve-se destacar ainda, a presença de diversas variedades das familias 960 e 1009, as quais apresentam como ancestral comum a espécie $V$. cinerea. Aliás, as nove primeiras variedades IAC dessa relação apresentam $V$. cinerea em sua genealogia, confirmando a contribuição dessa espécie para o aspecto considerado.

Como afirmado anteriormente, é provável que $V$. gigas assinalado na TABELA, seja de fato $V$. cinerea, o que se enquadra perfeitamente no que se discutiu.

Na Figura 14, observa-se a genealogia da variedade IAC 1565-2/Carolina onde se encontraram 3/64 vindos de 'Moscatel de Hamburgo' e 9/128 de 'Italia', mas deve ser considerado que 'Moscatel de Hamburgo' é progenitor de 'Italia'.
Aparentemente, a filosofia de trabalho do pesquisador Santos Neto, que efetuou a maior parte dos cruzamentos aqui analisados, era de fugir ou de distanciar-se o quanto mais de qualquer chance de endogamia. Isso fica bem nítido sobretudo quando se examina genealogias como as de 'Patrícia' (Figura 10), 'Yole' (Figura 11), 'Grão Mogol' (Figura 12), 'Marília' (Figura 13) e 'Carolina' (Figura 14) nas quais se podem contar até sete espécies diferentes.

O estudo das progênies fica um pouco prejudicado em razão do pequeno número de plantas. Contaram-se 125 famílias, das quais 73 com apenas 2 plantas, 32 com 3 plantas, 12 com 4 plantas, 4 com 5 plantas, 2 com 6 plantas, $1 \mathrm{com}$ 7 plantas e 1 com 9 plantas.

De todas as progênies, dezesseis tiveram pelo menos uma planta que acabou sendo selecionada e lançada como nova variedade: IAC 21, 74, 324, 344, 502, 503, 506, 775, 842, $871,960,1355,1398,1565,1595$ e 1726.

Algumas progênies, como a IAC $393 \mathrm{e}$ IAC 394, embora não tivessem apresentado cultivar algum selecionado, mostraram boa participação na genealogia de diversas outras variedades.

As progênies IAC 1595 e 1726 tiveram suas duas únicas plantas selecionadas como novas variedades. O destaque, porém, fica para a progênie IAC 871, resultante do cruzamento entre 'Soraya' e IAC 544-14, que teve 3 de suas 4 plantas lançadas como novas variedades: 'Geni', 'A Dona' e 'Patrícia', sendo as duas primeiras sem sementes.

A análise da genealogia da progênie IAC 871 acaba mostrando, afinal, a síntese de todo o programa, pois reúne, de certa forma, as cinco variedades mais utilizadas nos 48 anos: 'Italia', 'Niagara Rosada', a própria 'Patrícia', 'Moscatel de Hamburgo' e 'Soraya', e ainda a 'Seibel $13.053^{\prime}$, a $12^{\circ}$ mais usada.

\section{CONCLUSÕES}

Baseado nos dados aqui analisados, podem ser selecionados com preferenciais num programa de cruzamento para uvas de mesa as seguintes variedades: 'Itália', 'Patrícia', 'Soraya', 'Cardinal', 'Roberta', 'Maria Rosa', 'Carolina', 'Moscatel de Hamburgo', 'Lígia', 'Angelina', 'Marília', 'Alphonse Lavallée', 'Traviư', 'Ezequiel', 'Grão Mogol', 'Yole', 'Geni', 'A Dona', 'Aurora', 'Piratininga' 'Maria', 'Iracema'. 


\section{REFERÊNCIAS BIBLIOGRÁFICAS}

CAMARGO, U.A.; DIAS. M.F. Identificação varietal de algumas videiras cultivadas no Rio Grande do Sul. 2. ed. rev. Bento Gonçalves: EMBRAPA/ CNPUV, 1986a. 45p. (Circular Técnica. 11).

CAMARGO, U.A.; DIAS, M.F. Identificação ampelográfica de videiras americanas e híbridas cultivadas na MRH 311. Bento Gonçalves: EMBRAPA/CNPUV. 1986b. 40p. (Circular Técnica, 12).

DAFERT, F.W.; LEHMANN, E. Resultado das experiências de aclimatação da vinha. In: INSTITUTO AGRONÔMICO. Coleção dos trabalhos agricolas. São Paulo: Cia. Industrial, 1895. v.1, p. 335-340.

HEDRICK. U.P. The grapes of New York. Albany, J.B. Lyon, 1908, 584p.

PASSOS, I.R. da S.; POMMER, C.V.; HAAS, M.G.; PIRES, E J.P.; TERRA, M.M.; FALCO, M.C. Obtenção de hibridos entre variedades apirenas de videira utilizando a técnica de resgate de embriôes. Revista Brasileira de Fruticultura, Cruz das Almas. v.14, n.2, p.215-220, 1992.

POMMER, C.V. Uva. In: FURLANI, A.M.C. (Ed.). O melhoramento de plantas no Instituto Agronómico. Campinas: Instituto Agronômico. 1993. v.1. p.489524.
POMMER, C.V.; BASTOS, C.R. Genealogia de variedades IAC de cana-de-açúcar: vulnerabilidade genética e necessidade de programas básicos de melhoramento. Pesquisa Agropecuária Brasileira. Brasilia. v. 19. n. 5, p.623-629. 1984.

RIBAS, W.C. Contribuição à ampelologia nacional. Il. Melhores variedades da coleção ampelográfica da estação experimental de São Roque, S.P. Campinas, Instituto Agronômico, 1973. 76p. (Circular, 28).

SANTOS NETO, J.R.A. A cultura da videira. O Agronómico. Campinas, v.21, n.512,p.67-108, 1969.

SANTOS NETO, J.R.A. Melhoramento da videira. Bragantia, Campinas, v.14, n.23, p.237-257. 1955.

SANTOS NETO, J.R.A. O melhoramento da videira no IAC. Ciência e Cultura, Sāo Paulo, v.23, n.6. p.700$710,1971$.

SOUSA, J.S.I. Mutaçôes somáticas na videira niagara. Bragantia, Campinas, v.18, p.387-423, 1959.

SOUSA, J.S.I. Uvas para o Brasil. São Paulo: Melhoramentos, 1969. 454p.

Enviado para publicação em 28.03.94 Aceito para publicação em 26.05.94 